http://doi.org/10.15359/ree.12-1.8

\title{
SEXUAL-I-HABLANDO, LAS REPRESENTACIONES SOCIALES DE LAS ESTUDIANTES DE EDUCACIÓN PREESCOLAR EN RELACIÓN CON LA EDUCACIÓN PARA LA SEXUALIDAD
}

\author{
Martha Sánchez López ${ }^{1}$ \\ Académica del CIDE-Universidad Nacional \\ Heredia, Costa Rica
}

Recibido 22 de noviembre 2006• Aprobado 14 de marzo 2007

\begin{abstract}
Resumen: La presente investigación aborda las representaciones que tienen las estudiantes de la carrera de Educación Preescolar de la División de Educación Básica (DEB), sobre el curso Educación para la Sexualidad, impartido en el IV nivel del Bachillerato, a partir de la información que tienen las estudiantes sobre la sexualidad y de las expectativas respecto a su práctica profesional en las aulas preescolares sobre la educación para la sexualidad, con el fin de reflexionar acerca del impacto en los niveles pedagógico y subjetivo de la propuesta metodológica y de contenidos. También, se sugieren estrategias didácticas áulicas y la consecuente incorporación de éstas de parte de las y los estudiantes en su práctica profesional.

La metodología utilizada, desde el enfoque cualitativo, consideró adecuada la realización de entrevistas en profundidad, así como de relatos inducidos que dieran cuenta de las vivencias en torno a la educación para la sexualidad las dudas, temores, práctica docente y las estrategias para abordar la temática, entre otros. La investigación obtuvo como resultados una propuesta en contenidos y método para este curso. Además, una Prueba de Oraciones Incompletas (TOI), diseñado para conocer la información general que manejan las y los estudiantes que se matriculan en él, respecto de: la sexualidad, educación para la sexualidad, la sexualidad de las mujeres, la sexualidad de mujeres adolescentes, la sexualidad de las niñas, la sexualidad de los varones, sexualidad de varones adolescentes y la sexualidad de los niños.
\end{abstract}

Palabras clave: Sexualidad, Educación para la sexualidad, Socialización de Géneros.

\begin{abstract}
Due to the current need for information and mainly formation towards the topic about sexuality, it becomes a necessity to learn about the representations that Preschool Education students have around the class taught by the DEB (Division de Educacion Basica); specifically it deals with contents, methodologies and strategies that will for sure influence placing into practice the knowledge acquired in the classroom.
\end{abstract}

Académica del CIDE, Universidad Nacional, Heredia, Costa Rica. Licenciada en Psicología, Universidad de Costa Rica. Máster en Educación con énfasis en Docencia Universitaria. Miembro de la Asociación Costarricense de Gineco Obstetricia y Medicina Infanto Juvenil ACOGOMIJ. Correo electrónico: martasanchezlopez@gmail.com, coapsi@racsa.co.cr 
The present investigation aboards the representations that Preschool Education students from the DEB (Division de Educacion Basica) have about the class «Sexual Education» that is taught in the IV bachelor level; departing from the previous information about sexuality that these students have and the expectations related to their field work (student practice in preschool classrooms) about sexual education. All of the previous information has the purpose to reflect about the impact in the pedagogical and subjective level that the methodological proposal and the contents themselves show.

The methodology used, from a qualitative point of view, considered adequate making profound interviews as well as taking into account stories that illustrate experiences for situations lived around sexual education for students sexuality, also the doubts and fears during their field work and the strategies to come over this issue, among others.

The investigation obtained as a result a proposal in content and method for this class. Also, it suggests educational strategies in the classroom coming from the professors in charge, and as a consequence the incorporation of these strategies by the students during their field work. Besides, a test with incomplete sentences (TOI), designed to know the general information that students who will take this class handle, related to sexuality, sexual education, women sexuality, women, adolescents and girls, as well as men sexuality, adolescents and children.

Key words: Sexuality, Sexual Education, Genders Socialitation.

\section{INTRODUCCIÓN}

La reflexión acerca de la práctica docente en las aulas; en el desempeño de las labores y en la relación con los estudiantes a nuestro cargo, sitúan a la docencia en una posición de suma responsabilidad ante los procesos de formación de los que somos parte.

La investigación "Sexual-i-hablando, las representaciones sociales de las estudiantes de Educación Preescolar en relación con la educación para la sexualidad", responde a los requisitos de los Trabajos Finales de Graduación de la Maestría en Educación con énfasis en Docencia Universitaria, ofrecida por la Universidad Nacional. La utilidad de ésta será manifiesta en la valoración del impacto en los niveles pedagógico y personal de la propuesta metodológica y de contenidos del curso Educación para la Sexualidad, impartido en el IV nivel del Bachillerato de la carrera de Educación Preescolar de la DEB.

Resulta relevante que de esta investigación se generen líneas de trabajo para las carreras en Educación, referidas a la temática de Educación para la Sexualidad, que redunden en estrategias para la promoción de la salud, y por ende, de mejoras en la calidad de vida.

Actualmente, no se cuenta con un estudio que haya sondeado las expectativas, actitudes

y conocimientos de las estudiantes respecto de este curso, por lo que es de mucho provecho, acercarse a su significado, en relación total con las historias personales de la vivencia de la sexualidad.

Con esta investigación, se beneficiarán las personas que forman parte de la DEB: docentes, estudiantes, egresadas y egresados de las carreras que se imparten y éstas se traducirán en un panorama más claro respecto a la metodología y los contenidos del curso de Educación para la Sexualidad.

Es importante resaltar que, como parte del rediseño curricular, la temática de este curso formará parte de uno nuevo, denominado "Educación para la salud y calidad de vida", y que sin duda tomará como insumos los planteamientos del que le precede y los aportes de esta investigación. 


\section{HACIA LA VIVENCIA DE LA SEXUALIDAD INTEGRAL}

Cuando hablamos de sexualidad, nos viene a la mente una serie de conceptos asociados al tema, como sexos, géneros, orientaciones sexuales y prácticas sexuales, entre otros, que de una u otra manera, llevan a reflexionar acerca de nuestra propia existencia y vivencia. Campos y Salas la consideran como:

...una de las dimensiones fundamentales de los seres humanos, ya que implica la integración de los aspectos biológicos, socioculturales, psicológicos y éticos, que definen y constituyen a la persona como sujeto sexuado, como hombre y como mujer, incluyendo sus condiciones genéricas (2002, p. 19).

Una dimensión tan amplia que hace que confluyan desde los aspectos biológicos hasta los de condición más humana, como el amor, el respeto, la confianza, la intimidad, la sensualidad, el erotismo, la libertad, que se visibiliza en las formas en que se relacionan las personas, tanto en el ámbito fraternal, paterno-filial y de amistad, como de pareja.

Si hablamos de integralidad, para acercarnos a una visión holística de la sexualidad, no debemos dejar de lado que cada vez que se menciona la temática, nos referimos a la historia personal, a la crianza, a los vínculos, a la educación, que ayudan a moldear las acciones cotidianas.

Al respecto, Giddens (1992) menciona que en los tiempos actuales, la vivencia de la sexualidad está siendo mediatizada por otros cánones y formas de relación entre personas; esto implica que las concepciones, los límites, las libertades, entre otros, son reinventadas, como un juego de ir y venir en una constante y continua negociación.

Precisamente, en la vida cotidiana es donde, como lo señala Sandoval (1997, p. 21), “...toma forma la vivencia subjetiva de las condiciones estructurales y la acción social de los sujetos. Allí se fragua el sentido común"; ese que hace que las representaciones de la realidad se vuelvan una modalidad particular del conocimiento, cuyo fin es elaborar comportamientos y comunicación entre los individuos.

Según el autor, estas modalidades se constituyen en pensamiento práctico en relaciones sociales, debido a que los contenidos y procesos de representación están motivados por las condiciones y contextos en los que surgen; en las comunicaciones por las cuales circulan y, por último, en las funciones para las que sirven dentro de la interacción con el mundo y con los otros.

Si se considera lo anterior, podríamos aseverar que las representaciones construidas en relación con la sexualidad, toman forma y contenido no sólo en las historias personales de la gente, sino en la contextualización histórica y social de las que somos parte. La funcionalidad de que la pregunta acerca de la sexualidad tenga cabida en los contextos actuales, nos habla de una nueva forma de representar las relaciones con los otros, la corporalidad, la comunicación, las condiciones entre los géneros, los derechos, que hacen que la vivencia y su significado se lean desde otros lugares.

Para Sandoval (1997), quien retoma a Fernández (1991), las representaciones desempeñan funciones, y esta es la manera de mostrar no sólo el carácter, sino la forma en la que se han configurado. A su vez, distingue diversas formas para reconocerlas y establece al menos cuatro: las funciones de comprensión, de valoración, de comunicación y de actuación.

Respecto a la primera, menciona que remite a lo cognitivo, lo pensable o lo impensable; la de valoración aduce que se encarga de calificar situaciones, emite juicios acerca de lo concebible 
o no concebible. En cuanto a la función de comunicación, posibilita la interacción, lo nombrable o innombrable; por último, la de actuación condiciona las prácticas, lo realizable o no realizable.

El anterior desglose respecto a las funciones, permite, de forma más simple pero no menos compleja, acercarse a comprensión de esas maneras de representar la realidad social, que se traduce en el quehacer de las personas en cuanto a sus conocimientos, actitudes y prácticas, en relación consigo mismas, con los otros, las otras y con el mundo, y situaciones de la vida diaria.

La Teoría de las representaciones sociales da la posibilidad de acceder a una forma de conocimiento social que trasciende las opiniones, imágenes y actitudes. Siguiendo a Jodelet, (1988), citado por Araya (2002), la representación tiene carácter creativo y autónomo, lo cual no se refiere sólo al objeto, sino que influye sobre el comportamiento del sujeto, gracias a un proceso de elaboración cognitiva y simbólica.

Podría decirse, según Araya (2002), que es una forma de interpretar y de pensar nuestra realidad, y nos ubica en un punto donde se unen lo psicológico y lo social, el cual se construye a través de experiencias personales, informaciones, conocimientos y formas de pensar que se transmiten mediante la tradición, la educación y la comunicación.

\section{LA SUBJETIVIDAD EN JUEGO: CONSTRUYENDO, DECONSTRUYENDO Y RECONSTRUYENDO ESQUEMAS}

La subjetividad es un proceso que se construye a lo largo de toda la vida, y las constituye como sujetos singulares, particulares y únicos. Para Martín-Baró (1986), la socialización es el conjunto de procesos psicosociales por los que el individuo se desarrolla históricamente como persona y como miembro de una sociedad. Supone la adquisición de un mundo, al pasar a formar parte de una realidad objetiva, material y social. A partir de esta realidad, compuesta por relaciones sociales, símbolos, valores y normas, se afirma la identidad personal como un producto histórico y relativamente estable, en combinación con las relaciones interpersonales.

Desde esta lógica, se le confiere un lugar a lo singular e irrepetible, y desde la Psicología, surge el interés por la representación subjetiva que pregunta por la vivencia, por los conceptos, los conflictos y las potencialidades, para citar algunas.

Este proceso comunicativo para el estudio de la subjetividad, tal y como lo menciona Tovar (2001): "Implica el pasar de una concepción epistemológica basada en la relación sujeto-objeto, a una concepción basada en la relación sujeto-sujeto (...) significa una relación donde cada quien conserva su identidad, la cual es mediada por el diálogo en el que se involucran las partes desde sus propios saberes respectivos" (p. 148-149).

Las formaciones subjetivas, parafraseando a la misma autora, son indicadoras de la calidad del cotidiano que el sujeto se representa y vivencia; la indagación de éstas conduce a profundizar en dinámicas del orden de las creencias, valores, estereotipos, normas, sentimientos que caracterizan la configuración subjetiva como una totalidad.

Ese conjunto de representaciones o formaciones subjetivas del que un sujeto da cuenta, pasan necesariamente por la vivencia del día con día, aunada a la historia, y sin separarse de la condición de género de la cual se es parte. Al respecto, Burin y Meler (1999) señalan que las representaciones tradicionales de lo femenino y lo masculino atraviesan por un proceso de crisis que genera profundas ansiedades, y las nuevas prácticas de vida se vinculan de forma estructural a nuevas subjetividades. 


\section{METODOLOGÍA}

Esta investigación se enmarca dentro del paradigma cualitativo, que se caracteriza, según Patton (1980), por estudiar las situaciones del mundo real en forma natural, sin manipular ni obstruir los procesos. Además, profundiza en los detalles y datos específicos.

La investigación cualitativa trabaja desde una perspectiva holística, donde el contacto personal del investigador con los sujetos o las situaciones de estudio se mantiene. Da cuenta de los cambios en los niveles socio histórico y cultural y, en consecuencia, al estar inmerso dentro de la investigación, no es posible mantener la objetividad. Este paradigma privilegia el estudio casuístico y permite la flexibilidad de los diseños de investigación.

El tipo de investigación que guió el presente estudio, es la hermenéutica profunda, que se refiere a la posibilidad de interpretar un texto desde la perspectiva del investigador, pero cuidando los detalles del contexto en el que se genera.

Para efectos de esta investigación y a partir de los datos recopilados tanto en el Test de Oraciones Incompletas como en las entrevistas y relatos, se procede a aplicar el modelo de las irritaciones, complementado con los cuatro niveles funcionales de las representaciones que describe Sandoval (1997).

La población estuvo constituida por las integrantes de dos grupos de Educación Preescolar: "Las linces" (2003) y "Las gatas" (2004). En ambos grupos, cuando se inició el curso, incluso antes de leer el programa respectivo, se les solicitó llenar las frases incompletas, de forma anónima, con fin de usarlas para efectos de valorar los conocimientos sobre la temática de la sexualidad, y con miras a que la información se constituyera en un insumo para una posterior publicación. Todas las estudiantes estuvieron de acuerdo.

Además, para complementar la información, se eligió a cuatro estudiantes, dos de cada grupo, respectivamente:

\begin{tabular}{|l|l|l|}
\hline Caso número 1 & 2003 & $\begin{array}{l}\text { Killa (luna en lengua quechua), quien como la luna se muestra con } \\
\text { su carácter, unas veces imponente y otras retraída. }\end{array}$ \\
\hline Caso número 2 & 2003 & Inti (sol en lengua quechua), con ideas definidas, fuerte. \\
\hline Caso número 3 & 2004 & $\begin{array}{l}\text { Atl (mar en lengua Náhuatl), quien tiene mucho de la fuerza y la } \\
\text { tranquilidad del mar }\end{array}$ \\
\hline Caso número 4 & 2004 & $\begin{array}{l}\text { Ratna (esmeralda en lengua filipina), clara y coherente cual verde } \\
\text { azul. }\end{array}$ \\
\hline
\end{tabular}

\section{Instrumentos utilizados para la recolección de la información}

\section{Test de Oraciones Incompletas}

Se diseñó un test de oraciones incompletas con nueve frases, para conocer información general respecto de la sexualidad, educación para la sexualidad, la sexualidad de las mujeres, mujeres adolescentes y niñas, así como la sexualidad de los varones, varones adolescentes y niños, que manejan las estudiantes que se matriculan en este curso. 


\section{Entrevista en profundidad}

Para detallar la información y conocer de cerca el discurso de las estudiantes, se hicieron dos entrevistas en profundidad, de casi dos horas, a dos estudiantes (Atl y Killa) de cada año. El fin era relatar, a partir de una guía temática, las vivencias en torno a la sexualidad y educación para la sexualidad, así como sus expectativas, sus temores, su práctica docente y sus estrategias para abordar la temática, entre otros.

\section{Relato inducido}

Se realizó a las cuatro participantes, con Atl y con Killa como un medio para ampliar la información obtenida en la entrevista, así como recabar algunos datos sueltos, específicamente en relación con el impacto del curso en el desempeño profesional. A las otras dos participantes, Inti (2003) y Ratna (2004), se les aplicó, para complementar las información obtenida con las entrevistas a las otras participantes; se les solicitó que, de forma escrita, relataran los aspectos que más les habían impactado, personal y profesionalmente, del curso.

\section{La representación sin censura}

\section{Información respecto de la sexualidad que manejan las estudiantes ${ }^{2}$}

Las Linces refieren concepciones acerca de la sexualidad ligadas a las relaciones entre personas; para algunas se torna importante mencionar que el ser de uno o de otro sexo marca una connotación diferente en la vivencia de la sexualidad.

Aducen otra concepción relacionada con lo natural y, por ende, no necesita más explicación, la sexualidad es algo de la naturaleza; es decir, si es natural debe vivirse como tal; sin embargo, esta supuesta naturalidad se muestra cargada de visiones de mundo que han sido atravesadas por ideas erróneas, creencias, mitos y estereotipos que nunca se cuestionan.

Otras frases conciben la sexualidad desde una dimensión integral: lo que hacemos día con día, compartir con los demás en diferentes aspectos (social, familiar, parejas); también como el desarrollo físico, emocional y psicológico de todas las personas, en donde la afectividad y las relaciones interpersonales aparecen como necesarias. Es importante señalar que a partir de un discurso como el anterior, a pesar de que nos puede parecer muy completo y hasta ideal, en muchas ocasiones, se queda justamente allí, en el plano de la palabra, sin acciones que modifiquen los conocimientos, las actitudes y las prácticas.

En concordancia con lo anterior, ellas, mujeres al fin, siguen reproduciendo una serie de estereotipos en relación con los roles del género femenino, tal y como lo mencionan: la sumisión, la eterna abusada, minimizada, cuestionada, sin importancia, irrespetada, que están ligados a la

\footnotetext{
Todas palabras expresadas por las participantes de la investigación, se destacan en letra cursiva..
} 
agresión y al abuso por la condición de género, lo cual debe cuestionar acerca de los parámetros desde los cuales ellas se permiten mencionarlos.

Además, señalan la funcionalidad de los cambios en el nivel fisiológico relacionado con el mito de la maternidad, diciendo que la sexualidad de las mujeres juega un papel importante en la procreación y la consecuente preparación para una vida de posibles embarazos, que incluye: crecimiento de mamas y menstruación, entre otros.

En otras palabras, la representación social que surge de ellas acerca de la condición de género femenino, las sitúa en una profunda dicotomía; por un lado, la necesidad de evidenciar un discurso casi desde lo socialmente deseable acerca de lo que debe ser la vivencia de la sexualidad (integral, plena, que involucra varias dimensiones) y por otro, la contradicción respecto de lo que para ellas es la sexualidad de las mujeres. Sin embargo, este aspecto da cuenta de la realidad vivida no sólo como mujeres, sino por las historias socialmente construidas a su alrededor.

En el caso de la sexualidad de los varones, ellas dicen que es más visual, desordenada, acelerada, algunas veces grotesca, simple placer, es más fácil, pervertida, abierta, superficial, importante, marcado o permitido por la sociedad, social, sencilla, libre y menos cuestionada. Es decir, hacen una clara distinción entre la forma en la que ellas conciben cómo es la sexualidad de los varones y de las mujeres, cayendo en los extremos y filtrándose en lo que a diario se representa y reproduce.

Como bien lo señala Vega (2004), los mandatos y mensajes sobre la masculinidad y la feminidad traen consigo implicaciones para las vidas y las subjetividades tanto de los varones como de las mujeres, y estas demandas, socialmente impuestas, se asumen de forma individual y colectiva.

En esto, juega un papel importante la educación para la sexualidad; al respecto mencionan que ha sido mal dirigida, mal enfocada en solo aspectos carnales y por lo general orientada al sexo, lo cual se refiere a que en nuestro sistema educativo se le confiere relevancia a aspectos de la sexualidad ligados a la biología, y por lo tanto, despersonalizados.

También señalan que no es algo malo o prohibido si se tiene buenas bases morales, o sea, previendo que independientemente de lo natural que sea, su vivencia está asida a valores, cánones, discursos o reglas sociales que, en última instancia, constituyen mecanismos de control.

Por último, respecto de las relaciones entre géneros y la orientación sexual, remiten en su mayoría la vivencia de la heterosexualidad como única opción: es un proceso normal y natural que le permite convivir con el sexo opuesto; sin embargo, alguna se permite puntuar la posibilidad de la homosexualidad: si es posible el contacto con un hombre, por qué no con una mujer, lo cual hace que esta aseveración se constituya más en una excepción que en la regla.

Las Gatas, por su parte, mencionan que la sexualidad reúne las diferencias físicas y emocionales presentes en el ser humano, que brinda sensaciones, es algo digno de vivir $y$ necesario para el ser humano, e involucra las características físicas y psicológicas que distinguen al hombre de la mujer y viceversa. Aunque no dista mucho del aspecto biológico, sí se constituye en una posición más abierta, que implica tanto la genitalidad, las relaciones sexuales (coitales), así como también las interpersonales, es decir, cuando nos relacionamos con otros sexos, conocimiento de nuestros órganos sexuales y su funcionamiento, el conocerme a mí misma y aceptar mi propio yo.

En cuanto a la sexualidad de las mujeres, ellas refieren que la feminidad añade un poco de dificultades a la misma; mencionan, entre otras cosas, el instinto femenino, la necesidad de estar en compañía de un hombre, aspectos físico o biológico (reproducción), aún es visto como un tabú, con límites, muy discreta, es muy compleja, más difícil que la de los hombres, reprimida. Cabe decir, que la forma en la que es concebida la sexualidad de las mujeres trae consigo un encargo para su 
vivencia, lleno de ataduras y de culpas; se percibe que, por el hecho de ser mujeres, biológicamente hablando, se está destinada a una vida sin libertad.

En relación con la sexualidad de los varones, la definen a partir de la heterosexualidad. Aunque alguna se permitió reconocer la homosexualidad como opción para relacionarse, se aduce que la sexualidad del varón se reduce al instinto, y por lo tanto, se refuerzan los estereotipos en cuanto a roles de género. Surge la pregunta acerca de qué dirán los varones si escucharan a estas mujeres haciendo tales aseveraciones, sobretodo, porque es harto sabido que estos resultados tendrían otra connotación si se hubieran construido en grupos mixtos.

En relación con lo que les han enseñado acerca de la sexualidad, aseguran que es poco, y que hay que ser conservador, que es natural, que todos la poseemos, que debe existir y aprobarse entre dos seres, que no es malo, que se puede vivir con responsabilidad, que es demasiado viciado y hasta concebido como vergonzoso. En sus frases, queda muy clara la visión de la sexualidad como un asunto vedado, del que casi no se habla, pero que puede vivirse con responsabilidad. Algo así como que: es parte de mí, pero que no la conozco lo suficiente, aunque debo ser recatada, porque si sé mucho se volvería algo inadecuado y si admito que manejo información también, los valores morales me protegen de alguna forma en las experiencias que se vivan.

Estas son las frases sobre la sexualidad, y que, permeadas por mitos, estereotipos y tabúes en relación con las mujeres, varones, orientaciones sexuales, la educación para la sexualidad, entre otros, tienen las estudiantes. Ambos grupos no distan en opiniones, y esto clarifica la forma en que las representaciones construidas sobre la sexualidad, posibilitan dictar juicios sobre lo que se percibe como concebible o no en la vida cotidiana.

La forma en la que ellas piensan y construyen su propia de visión de mundo no es gratuita; las historias de crianza particulares, conjugan a múltiples protagonistas como la familia, el barrio, la escuela, el colegio, las amistades, que cotidianamente forjan huellas imborrables.

Conozcamos cuatro historias. Las de Atl, Killa, Ratna e Inti, que cuentan la vivencia de su sexualidad.

\section{LAS HUELLAS IMBORRABLES: HISTORIA FAMILIAR, MITOS, PREJUICIOS, CREENCIAS, TABÚES, ESTEREOTIPOS}

\section{¿Cómo fueron criadas?}

Esta es una de las preguntas que nos hacemos en relación con la actuación de las personas y el condicionamiento de sus prácticas cotidianas. En el mundo de la docencia nos percatamos de las particularidades que convergen en un solo lugar: "Yo he tenido una educación diferente a la del resto, totalmente. En mi casa siempre nos han hablado abiertamente, sin ningún tapujo y así es mi forma de ser".

Killa anota al respecto: "...yo soy muy abierta, yo hablo mucho de este tema con mis papás, es que soy hija única y eso es diferente. La cuestión que surge es si esta apertura. Pretenderíamos reconocer si las formas de crianza se trasladan los ámbitos del aula universitaria (entre compañeras) y escolar (con sus estudiantes).

Por otro lado, la voz de Inti asevera con tono enérgico: "mi pensamiento está dirigido por principios bíblicos y me parecía chocante el escuchar muchos comentarios que a mi criterio no 
están bien”. Inti es de un pensamiento rígido en cuanto a información que no concuerde con sus preceptos religiosos. Ligado al relato de las otras compañeras, nos preguntamos, de qué manera se permea la práctica docente a partir de pensamientos y creencias religiosas o de otro tipo de valores o cánones de vida. ¿Cómo desprenderse de estos aprendizajes y no filtrarlos en la práctica profesional, sobre todo considerando la diversidad de que somos parte y que hace un llamado a cuán respetuosos y tolerantes seamos en diferentes ámbitos?

No se debe dejar de lado el papel de las familias en este entramado de relaciones, de pautas de comportamiento y de actitudes, ya que tiene una lógica de funcionamiento. En un primer momento la familia educa, y luego la escuela, a través de la figura del docente, que es bien sabido, está constituida, en su mayoría, por mujeres. Es decir, con los relatos de las participantes nos percatamos de que ellas reproducen lo que en aprendieron en el seno familiar y con sus propias maestras.

\section{¿Cómo se comunican y se relacionan con la familia, con la gente cercana?}

Tanto Atl como Killa tienen historias distintas en cuanto a sus lugares de origen, sus costumbres y su composición familiar. Atl menciona que su vida como buena citadina ha sido excéntrica, sobre todo si se compara con las historias de sus compañeras de grupo. Ella dice: "...yo me he relacionado con otro tipo de gente, fiestas, yo fumaba. La clase...no digo que sea elitista pero que sé yo, salir y tomarme 4 whiskys, no es problema y no tengo que pensar en que si salgo se me acaba la plata de los pases.

Aun así, reconocen que estando en la carrera de Preescolar, tuvo una especie de regresión o una necesidad de infantilizarse, y señala:

Yo le decía a mi mamá : ¿por qué no me vas a recoger a tal lugar, como lo hacían con las otras? Y mi mamá: pero qué te pasa yo no tengo que cuidarte nada, no salís de madrugada de los bares y te venís en taxi? Yo quería que me chinearan como a todas.

Es interesante la necesidad de Atl de verse protegida, o al menos tratada igual que sus compañeras: Recordemos que ella estuvo primero en otra carrera y luego comienza la de Educación, donde la mayoría son mujeres y, en este caso, menores que ella. Surge la interrogante sobre la libertad de la que goza esta muchacha, y su necesidad de recibir protección materna.

En el caso de Killa, la situación es diferente, debido a que es hija única y viene de una zona alejada de la Universidad; lo que la obliga a vivir en un apartamento con otras amigas; en cuanto a esa relación:

A ellas yo les cuento, el año pasado después de la clase, todo se los contaba, porque aunque uno supiera algo, siempre se aprendía algo diferente, de lo que usted daba y lo que decían las compañeras.

Killa dice que tanto con su mamá como con su papá habla de todo, en especial sobre sexualidad; pero que su mamá es menos abierta y su papá tiene un pensamiento muy machista: “...yo les digo la sexualidad abarca todo, abarca toda la vida, pero a mi mamá le da vergüenza en cambio mi papá comparte eso conmigo". 
De su mamá dice: “...yo hablo con mi mamá, qué se yo de los homosexuales y ... mami ahí déjelos que vivan sus vidas, y yo le digo que son iguales que nosotros; y ella no, no acepta que está en otro chip. Al referirse a la forma en la que su madre piensa, asegura que aún cuando conversan de todo, algunos temas como la orientación sexual, no son tratados abiertamente.

Menciona que con su novio habla mucho de sexo, porque en la casa de él de eso no se habla, y que en su noviazgo todo está bien en este sentido. En la entrevista, Killa asegura que, aunque ella es muy abierta respecto la tema de la sexualidad, cree que esto le ha permitido tener una relación satisfactoria con su novio, sin tener que llegar a la genitalidad, porque ambos consideran que es una dimensión para la que todavía "no estamos preparados".

\section{¿Cómo fue su educación?}

\section{La información dada por terceros}

Con mucha frecuencia, creemos que la mayoría de la información que manejamos, ha sido obtenida, principalmente, en la familia y en la escuela; si bien esto no deja de ser cierto, existe mucha gente alrededor para darnos más detalles que no necesitamos, para aclararnos o confirmarnos información de nuestro interés. Killa nos cuenta:

Más chiquitilla, me acuerdo que una vez fui donde una tía y mi prima que tenía como 15 me dijo, sabe ¿cómo se hacen los bebés? Y yo, no, supe que decir; entonces me explicó todo. Que la semillita y que el papá y la mamá... yo tenía como 4 años y le dije Ah!!... y me di la vuelta para dormir.

Es evidente que muchas veces ocurre que hay información que los niños están buscando, pero otras veces como adultos/docentes, entramos en la angustia de lo que deben conocer las niñas y niños; sin embargo, no nos percatamos o sacamos un momento para preguntar si realmente quieren saber sobre este tema.

También Killa nos habla de su relación con otras personas, en específico, con varones adolescentes mayores que ella:

Yo siempre fui muy desarrollada, los hombres son más lerdos, fui muy precoz, y yo ¿por qué yo?, siempre me salí de lo normal, yo era más grande y en sétimo me tiraban pelota los de quinto y yo como si nada, y ellos... seguro ¿qué le pasa a esta?

De alguna forma esta, es la negociación cotidiana, por la que tanto mujeres y como hombres pasan, en todas las edades, pero que se exacerba en la adolescencia. Es más, Kika dimensiona las prácticas sexuales, como el besarse, de una manera distinta “...yo no me besé con nadie hasta como en octavo, yo no fui de apretarme con todo mundo, solo por sentir rico, yo tenía que sentir algo.

La adolescencia genera muchas angustias, sobretodo por los acelerados cambios en el nivel corporal, que no coinciden con el desarrollo o los cambios en el plano psicológico y de pensamiento. Desde este punto, es necesaria la revisión de las experiencias personales que cada uno tuvo como adolescente, para intentar acercarnos a losalumnos con una escucha más sensible. 
En casa y en familia

Para Killa, la primera vez que le hablaron de su cuerpo fue en su casa: "...mi mamá me explicó lo de la menstruación y ya, cuando me vino yo le dije y también a mi papá y me dijo: ah! Ya es mujer, y yo cómo yo soy desde que nací. Hubo alguna información desde el ámbito de lo biológico que no clarificó la situación. Los padres y madres hacen lo que, según ellos, está bien y brindan información para la que no tienen el conocimiento suficiente o no están preparados; esto no quita la posibilidad de que ellos se instruyan, al igual que sus hijas e hijos, su visión acerca de la sexualidad.

Para Atl, su educación ha sido muy abierta: “...yo tengo varias hermanas mayores y a todas, mami siempre nos explicó todo y cómo cuidarnos, más bien yo le decía: Mami, yaa...! Ambas son extremos: una sin información y deseándola, y esta última, deteniendo a una madre explícita. ¿Cuál es, entonces, el punto de equilibrio en una temática que requiere ser abordada con mucha naturalidad, pero sin violentar procesos?

A ella, tanto la abuela como la madre, siempre le enseñaron a defenderse y a no dejar que nadie, y menos un hombre, nos tratara mal. Atl describe esta relación:

He vivido desde que tengo memoria, en un hogar donde lo que prevalece es un matriarcado, por esta razón y muchas otras, siento que mi visión del mundo en general, es diferente de la que prevalece en la aulas de educación preescolar, en las que todas tienen la idea de que para ser buena maestra hay que tener una doble moral, en cuanto a lo que se finge ser en el aula y en cuanto a lo que verdaderamente somos!

Y sigue señalando:

Yo comencé estudiando Topografía, pero luego me cambié porque necesitaba de una carrera en la que tuviera contacto con la gente, devolverle algo, que deje algo en los demás. Además de que me gusta el trabajo con los niños, porque son abiertos, espontáneos, claros.

Al inicio cuando entré, era como ¿qué estoy haciendo aquí? Con este poco de chiquitas, es que imagínese, tres años menores que yo. Es que vienen de hogares donde siguen siendo chiquitas, como mientras me caso, y yo no comparto eso, no es mi ideología.

Esta aseveración muestra cómo se concibe la docencia con un papel dicotómico: por un lado, el deber ser, que implica una vida modelo, y por otro, el ser mujer y humana, que tiene que ver con la vida y las experiencias buenas o malas, agradables o no, de cada una.

Para el caso de Killa, el noviazgo pasa por la formalidad y la educación recibida en la familia; ella menciona:

Yo tengo novio hace 5 años, es una relación formal, mi papá es muy celoso, me decía que los hombres buscan mujer, no novia, que los hombres piensan solo en tener relaciones con las mujeres. Pero yo nunca lo iba a hacer, era como una ideología familiar, yo la eduqué bien. Me decían cuídese, pero nada más. 
Quizá la mejor forma de ilustrar lo que los padres identifican como haber dado buena educación para la sexualidad a los hijos e hijas, es el famoso "cuídese", como si esta palabra implicara, en sí misma, todos los mandatos de lo que se debe o no hacer, a partir de los cánones familiares.

En la escuela, recuerdos muy vagos

¿Qué recuerdo de la escuela? Dice Atl: “¡diay! lo clásico, lo más biológico, lo del aparato reproductor, pero no había tanto morbo, ahora hay más morbosidad".

Killa anota que, dado que ella viene de una zona rural, los roles de género están marcados, y filtrados por el machismo. Recuerda que, en la escuela, los hombres recibían clases de agricultura y las mujeres se quedaban limpiando el aula.

Enfaticemos aquí la importancia de la escuela en la formación de las personas, y que muchas veces, actitudes o formas de comunicación llegan a moldear sistemas de creencias y pensamiento.

¿Y en el cole?

Según las jóvenes, la información recibida sobre sexualidad se da en las clases de Biología; ahí se les explica sobre el cuerpo humano y las funciones de los aparatos reproductores femenino y masculino; pero desvinculada de una integralidad que sus experiencias, cambios corporales, sensaciones, temores, dudas o las posibilidades de promover la salud sexual desde los componentes del autocuidado y el cuidado mutuo.

La otra parte de la vivencia del colegio, y no menos importante, en cuanto a la sexualidad, está mediatizada por el grupo de pares, el cual ejerce un papel de modelador y generador de directrices en relación con la información y los comportamientos entre iguales. Atl dice “...en realidad, todo siempre lo supimos desde la casa y en el colegio, pues más era el interés por saber qué hacía cada una.

Killa dice que recibió datos durante su décimo año en “...Biología, pero uno sabe algunas cosas, uno sabe por los comentarios de los compañeros; es decir, hay mucha información que circula entre amigos; habría que revisar la veracidad de ésta, ya que muchos contarán sólo con esta información para el resto de sus vidas.

Lo anterior, hace un llamado a la ausencia de formación en los años de escuela y de colegio. Considero que, desde el mundo adulto, genera mucha angustia reconocer que no sólo los niños y los jóvenes se preguntan sobre el tema, sino que además, no estamos preparados para responder en forma adecuada a sus interrogantes, y la fórmula que se ha seguido para abordarlo, ha sido desde la depersonalización, en la clase de Biología, donde los aparatos y órganos son vistos como órganos aislados, que no son parte de un cuerpo completo y del ser humano integral.

\section{Ganancias para la vida}

¿Qué deja el curso para sus vidas? Las anotaciones van en muchos sentidos. Atl comenta, “...uno en ocasiones piensa que ya no existen aquellas ideologías, a mi parecer ortodoxas, tales 
como tener sexo hasta que me case; o del odio hacia la gente homosexual y no digo que yo sea una persona libre de perjuicios, porque sí los tengo. En otras palabras, la posibilidad de escuchar distintas opiniones y saber que todas coexisten, es parte de las experiencias que se proveen. Y prosigue con sus señalamientos:

Me fui percatando de la importancia de impartir este curso en la carrera de preescolar y una de las razones que pude apreciar fue la de que algunas compañeras se sentían incómodas al tener que abarcar y hablar acerca de sexo, a unas solo les faltaba pedir una libreta, y empezar a pedir consejos sexuales (las más inexpertas); otras mencionaban que se sentían extrañas cuando veían a algún bebé erecto, otra de que jamás permitirá a sus hijas tener relaciones en el noviazgo.

También, reconoce que existe información que debe ser aclarada, como:

Gran cantidad de terminologías que yo desconocía, por ejemplo la diferencia entre SIDA y VIH, lo de período del ventana, la afectividad en la sexualidad. Siento que he logrado respaldar mis conocimientos previos en cuanto a la sexualidad y que he reforzado otros aspectos que se deben de tomar en cuenta a la hora de trabajar con infantes.

Ratna, por su parte, cuenta que la experiencia del curso la insertó en “...Un mundo prohibido, donde las preguntas nunca eran respondidas y por fin todos aquellos pensamientos que por miedo, por vergüenza, creencias religiosas entre otros, y me hacían sentir una mala persona, se abrieron ante mis ojos, dejándome ver una realidad que todos vivían, me liberé de todos esos tabúes que me habian frustrado por años.

Cuesta imaginarse lo que esta joven señala, acerca de no contar con espacios, ni en la familia ni en los centros educativos, para hablar abiertamente sobre sexualidad. Esto confirma la ventaja de contar con un curso, en el nivel universitario, que enfatice en estas temáticas y que les dé la posibilidad de escuchar, con otra sensibilidad, los relatos de sus compañeras, que implican miedos, dudas, vergüenzas y experiencias positivas.

\section{La maestra, la niña, la "teacher" y la mujer sexuada}

El impacto del curso en relación con la carrera, con el ser docente y con la práctica profesional

Uno de los aspectos que las participantes rescatan, es el reconocimiento de las diferentes maneras de ver el mundo, y en especial, ligado al tema de la sexualidad. Parece mentira, pero después de estar casi cuatro años juntas, hasta este momento cuando se les da la oportunidad de conocer su vivencia como seres humanos. Atl dice al respecto,

Creo que mi visión del resto del grupo cambió con respecto a diversos factores, por ejemplo, en ocasiones uno juzga a las personas que piensan o son diferentes a uno, pero escuchándolas hablar, pude ver que todas las cosas tienen una razón de ser y que la manera en la que hemos sido criadas influye demasiado en nuestra personalidad, 
convicciones, manera de relacionarnos, etc. aunque ya seamos adultas y podamos discernir entre lo que nos parece o no, siempre vamos a quedar marcadas por esas huellas familiares.

Todas las participantes mencionan la importancia de la metodología participativa como coadyuvante en la dinámica de la clases, en particular, porque el aporte de todas fue enriquecedor, dada la cantidad de ejemplos de la vida cotidiana y de sus experiencias.

Para Killa, resulta de sumo interés el hecho de que al hablar de la propia sexualidad, se puede aprender cómo es la de los demás; lo dice de forma pintoresca:"... para mí fue importante confrontar todo lo que he vivido, es como al caer en el chayote de cómo ha sido la propia sexualidad y la de los niños". Al construir el concepto acerca de la sexualidad, en el nivel personal, de facto este va a ser trasladado al profesional, ya que se tiene un mejor criterio para conversar sobre el tema.

Atl señala que con el curso sobre sexualidad se rescata:

La importancia de que los niños y niñas aprendan a conocerse y a aceptarse a sí mismos y a aprender a relacionarse los unos con los otros. Además la educación sexual afectiva es una forma de potenciar responsabilidades y formas de tomar decisiones correctas, por medio de actitudes y comportamientos positivos, lo cuales deben irse tratando desde que los niños están pequeños.

Se valora la educación para la sexualidad como un componente vital para la formación de niñas y niños. Por último, Ratna menciona que las ganancias para la vida profesional han pasado a ser primero en el plano personal:

El poder hablar de nuestras vidas sexuales con tranquilidad, de entender que ésta no es mala, que lo que cargamos dentro de sí, son un montón de tabúes, de poder crear medios para hablarle a los niños, sin vergüenza y a la vez con los mejores términos, tomando en cuenta sus necesidades, intereses, edad y dudas, indudablemente como docente me ayudó demasiado.

Todas señalan la importancia del aprendizaje de conceptos nuevos, pero de igual forma, reconocen las diferentes perspectivas que se tienen acerca de la sexualidad.

De acuerdo con la Teoría de la representaciones sociales, los relatos denotan cuatro funciones: desde el nivel de la compresión hay mucha información errónea y la valoración de las formas de crianza. La función comunicativa, que atraviesa la labor docente se transmite tal cual, sin censura alguna.

Otra función no menos importante, es la de actuación; como ellas mencionan, las lleva a realizar, en muchas ocasiones, una vida como docentes y otra como personas: mujeres de carne y hueso.

\section{RECOMENDACIONES}

Estas son las voces de las Linces y las Gatas, la de Atl, Killa, Inti y Ratna. Ellas quieren que comunique algo a partir de su experiencia, escuchemos... 
Creo que es importante incluir la perspectiva masculina (Atl).

Debería de haber más cursos de estos y abrirlo a otras carreras; para tener más opiniones, empezando por la de los hombres (Killa.)

El mundo de las carreras de Educación, y en especial en Preescolar, es femenino, por lo que todo lo que aquí se construya tiene esta mirada. ¿Qué pasa cuando aún el reconocimiento de la diversidad en términos de la sexualidad, se construye desde la visión de mundo de las mujeres? Esto no significa que no se hable de la perspectiva masculina, sino que se aborda desde una mujer (la docente), y con un auditorio exclusivo de mujeres.

La recomendación para el curso optativo, sería que se abra a otras carreras, al otro género, a otros pensamientos, para no seguir perpetuando la idea de que somos las mujeres las encargadas de educar.

El curso tiene que estar en el nivel de diplomado y que no sea optativo sino de los fundamentales (Atl).

El curso es importante, porque se comentan casos diarios y a uno le sirve, porque generalmente termina uno dando clases en escuela y a veces en sexto y de dónde me agarro (Killa).

Se solicita que el curso se imparta en la formación inicial en el Diplomado, como parte del plan de estudios, y no como un curso optativo. Sobre todo, porque una vez que las estudiantes se han egresado, o cuando comienzan a laborar, no es precisamente en Educación Preescolar donde trabajan, sino que asumen responsabilidades en I y II ciclos de la Educación General Básica.

Las estudiantes rescatan el hecho de que la relación teoría-práctica va de la mano, y muchas situaciones referidas a la temática de la sexualidad se presentan desde los primeros niveles de la carrera, en su contacto con estudiantes a partir de los cursos prácticos y teórico-prácticos; estos no han tenido el asidero suficiente, tanto en contenidos como en metodología, para saber si el abordaje realizado fue el idóneo.

Por último, la cantidad de temas propuestos es muy extensa. El curso llega a tocar una temática que, por su amplitud, va a enlazarse con múltiples temas de la vida, a partir de la consigna de que todos somos seres sexuados y distintos; de ahí que la controversia surge desde el inicio, al hablar de sexualidades y no de una sexualidad.

Recapitulando, las sugerencias, en términos generales, se resumen así:

- La importancia de la experiencia grupal y la participación como generadora de aprendizajes significativos, debido a que el curso, en el nivel personal, pasa por la necesaria revisión y reflexión de la vivencia de la propia sexualidad.

- $\quad$ El docente a cargo del curso debe estar dispuesto a correrse del lugar del saber y lograr que la empatía sea uno de los ejes que guíen el desarrollo del curso.

- La metodología participativa da excelentes aportes para desarrollar estrategias de aprendizaje que permitan la incorporación y revisión (metacognición) de los elementos principales de ésta, para su aplicación posterior.

En definitiva, aunque las estudiantes rescatan el hecho de salir con un concepto más amplio acerca de la sexualidad, los conocimientos, actitudes y prácticas (CAP), requieren no sólo del 
tratamiento del tema como eje transversal en la carrera, sino de mayor investigación y evaluación, que den cuenta de la experiencia del curso en las vidas profesionales de las estudiantes.

\section{REFERENCIAS}

Araya, S. (2002). Las representaciones sociales: Ejes teóricos para su discusión. Cuaderno de Ciencias Sociales 127, 1-82.

Burin, M., Meler, I. (1999). Género y familia. Argentina: Editorial Paidós.

Campos, A. y Salas, J. (2002). El placer de la vida: sexualidad infantil y adolescente: su pedagogía a cargo de personas adultas. San José, Costa Rica: Lara Segura.

División de Educación Básica. (2002). Plan de estudios en pedagogía con énfasis en educación preescolar. Heredia: Universidad Nacional.

Gidden, A. (1992). La transformación de la intimidad: sexualidad, amor y erotismo en las sociedades modernas. Madrid: ediciones Cátedra.

Martín, I. (1986). Acción e ideología. Sicología social desde Centroamérica. San Salvador: UCA Editores.

Patton, M. (1980). Qualitative evaluation and Research mettods. USA: SAGE publications. Compilación: Antología Paradigma Cualitativo. Maestría en Educación.

Sandoval, C. (1997). Sueños y sudores de la vida cotidiana: Trabajadores y trabajadoras de la maquila y la construcción en Costa Rica. San José, Costa Rica: Editorial de la Universidad de Costa Rica.

Tovar, M. A. (2001). Psicología social comunitaria. Una alternativa teórico metodológica. México: Editorial Plaza y Valdés.

Vega, M. (2004). Género y Salud. Hacia una atención integral de las y los adolescentes. San José, Costa Rica: CCSS-PAIA-UNFPA. 